\title{
CAVEOLIN-1 expression in brain metastasis from lung cancer predicts worse outcome and radioresistance, irrespective of tumor histotype
}

\author{
Eleonora Duregon ${ }^{1, *}$, Rebecca Senetta ${ }^{5, *}$, Alessandra Pittaro ${ }^{5}$, Ludovica Verdun \\ di Cantogno ${ }^{5}$, Giulia Stella ${ }^{2}$, Pierpaolo De Blasi ${ }^{3}$, Michele Zorzetto ${ }^{2}$, Cristina \\ Mantovani ${ }^{4}$, Mauro Papotti ${ }^{1}$ and Paola Cassoni ${ }^{5}$ \\ ${ }^{1}$ Department of Oncology, University of Torino at San Luigi Hospital, Orbassano, Turin, Italy \\ ${ }^{2}$ Laboratory of Biochemistry and Genetics, Pneumology Unit, Department of Molecular Medicine University and Fondazione \\ IRCCS Policlinico San Matteo, Pavia, Italy \\ ${ }^{3}$ University of Torino and Collegio Carlo Alberto, Torino, Italy \\ ${ }^{4}$ Department of Oncology, Radiation Oncology, University of Torino, Italy \\ ${ }^{5}$ Department of Medical Sciences, University of Torino, Italy \\ * These authors have contributed equally to this work \\ Correspondence to: Paola Cassoni, email: paola.cassoni@unito.it \\ Keywords: Caveolin 1, brain metastasis, non-small-cell lung cancer, radiotherapy \\ Received: April 10, $2015 \quad$ Accepted: July 16, $2015 \quad$ Published: July 22, 2015
}

This is an open-access article distributed under the terms of the Creative Commons Attribution License, which permits unrestricted use, distribution, and reproduction in any medium, provided the original author and source are credited.

\section{ABSTRACT}

Brain metastases develop in one-third of patients with non-small-cell lung cancer and are associated with a dismal prognosis, irrespective of surgery or chemoradiotherapy. Pathological markers for predicting outcomes after surgical resection and radiotherapy responsiveness are still lacking. Caveolin 1 has been associated with chemo- and radioresistance in various tumors, including non-small-cell lung cancer. Here, caveolin 1 expression was assessed in a series of 69 brain metastases from non-small-cell lung cancer and matched primary tumors to determine its role in predicting survival and radiotherapy responsiveness. Only caveolin 1 expression in brain metastasis was associated with poor prognosis and an increased risk of death ( $\log$ rank test, $p=0.015$ ). Moreover, in the younger patients (median age of $<54$ years), caveolin 1 expression neutralized the favorable effect of young age on survival compared with the older patients. Among the radiotherapy-treated patients, an increased risk of death was detected in the group with caveolin 1-positive brain metastasis (14 out of 22 patients, HR=6.839, 95\% CI 1.849 to 25.301, Wald test $p=$ $0.004)$. Overall, caveolin 1 expression in brain metastasis from non-small-cell lung cancer is independently predictive of worse outcome and radioresistance and could become an additional tool for personalized therapy in the critical subset of brainmetastatic non-small-cell lung cancer patients.

\section{INTRODUCTION}

Brain metastasis from non-small-cell lung cancer (NSCLC), the leading cause of cancer-related death worldwide, develops in $20-40 \%$ of patients $[1,2]$ at a relatively earlier stage compared with other primary cancers. It is associated with a dismal prognosis, with a median survival of one month in untreated patients.
Other than supportive care with corticosteroids, surgery, whole-brain radiation therapy (WBRT), stereotactic radiosurgery and chemotherapy, either alone or in various combinations, are valuable options for these patients [3]. Although WBRT is still the most effective treatment following surgery [3], the overall survival is about five months after WBRT, and only $5 \%$ of patients survive one year $[4,5]$. Prognostic factors affecting survival include 
Table 1: Clinical and pathological features of 69 patients with NSCLC metastatic to the brain analyzed for Cav1 expression

\begin{tabular}{|l|l|}
\hline Parameter & \\
\hline M/F ratio & 3.31 \\
\hline Age, mean (years) [range] & $64[35-80]$ \\
\hline Number of brain metastases & $\begin{array}{l}\text { single: } 49 \\
\text { multiple: } 20\end{array}$ \\
\hline Histotype & $\begin{array}{l}\text { adenocarcinoma: } 38 \\
\text { squamous cell carcinoma: } 14 \\
\text { neuroendocrine carcinoma: } 7 \\
\text { large cell carcinoma: } 10\end{array}$ \\
\hline Type of radiotherapy & $\begin{array}{l}\text { adjuvant : } 22 \\
\text { cytoreductive: } 9\end{array}$ \\
\hline Disease status (lost to FU: 1 ) & $\begin{array}{l}\text { NED/DOC: } 14 \\
\text { DOD: } 57\end{array}$ \\
\hline Median overall survival (months) & $14[1-84]$ \\
\hline
\end{tabular}

age, performance status, the number, locations, and sizes of brain metastases, the presence or absence of symptoms and/or mass effect, suitability for surgical resection, and availability (or not) of options for controlling extracranial disease [6]. However, pathological tools for selecting patients who may benefit from surgical resection of brain metastasis, for predicting outcomes after surgical resection and for predicting radiotherapy responsiveness are lacking.

Among various prognostic and predictive markers, caveolin 1 (Cav1) has been associated with chemoresistance in various solid tumors, including NSCLC [7, 8, 9], and with radioresistance in pancreatic $[10,11]$ and colon cancers [12]. Cav1 is an essential structural constituent of caveolae, acting as a multifunctional scaffolding protein with multiple binding partners. Its ability to interact with numerous proteins makes it a central cellular signaling molecule. Cav1 organizes signaling complexes at the inner plasma membrane to activate a variety of cellular events and has been implicated in neurodegenerative diseases, diabetes mellitus, senescence and oncogenesis [13]. In cancer, Cav1 is involved in cellular transformation, tumor growth, cell death and survival, multidrug resistance, angiogenesis, cell migration and metastasis [14]. A biphasic role of Cav1 in signal transduction and cancer has been thoroughly documented. It acts as a tumor-suppressing protein during the early stages of cancer progression, but once it becomes upregulated during more advanced stages of disease, it gains an oncogenic function, which contributes to an aggressive and metastatic phenotype [14]. In agreement with this oncogenic potential, in vitro Cav1 knockdown of NSCLC cells has been shown to result in inhibition of cellular proliferation [15], whereas Cav1-overexpressing NSCLC cells, as well as anoikis-resistant cells, exhibit enhanced metastatic activity [16]. Meta-analysis of NSCLC patients [17] has revealed that although the Cav1 level is significantly lower in cancerous tissues than in non-neoplastic lung tissues, Cav1-expressing NSCLC patients have a higher risk of death and reduced progression-free survival, consistent with a role of Cav1 in tumor progression. We have previously described the differential expression of Cav1 according to NSCLC histotype and its increased expression in brain metastasis [18].

To the best of our knowledge, neither a hypothetical prognostic role of Cav1 expression in brain metastasis from NSCLC nor its relationship with response to radiotherapy has been assessed. Therefore, we evaluated the role of Cav1 in predicting survival and radiotherapy responsiveness in 69 patients with NSCLC with brain metastasis. We found that a) Cav1 expression in brain metastasis was related to poor prognosis; b) in younger patients, Cav1 expression in brain metastasis resulted in an increased risk of and neutralization of the favorable impact of young age on survival; and c) Cav1 positivity in brain metastasis was predictive of radioresistance.

\section{RESULTS}

\section{Clinico-pathological data and Cav1 protein expression}

The main clinical and pathological features of the whole series of 69 cases are summarized in Table 1. A total of 38 cases $(55 \%)$ were adenocarcinomas. Cav1 expression in primary lung carcinoma and matched brain metastasis exhibited a significant association (chi-square test $p<0.001)$. Overall, $16(23 \%)$ primary tumors and 28 brain metastases (41\%) expressed Cav1, with mild to strong membrane or cytoplasmic staining of neoplastic cells (Figure 1). Fourteen negative primary tumors shifted to Cav1 positivity during metastasis, whereas two positive lung carcinomas lost Cav1 expression in the brain metastatic tissue (Table 2). Cav1 brain metastasis expression and histotype were significantly associated (chi-square test $p=0.043$ ) because all of the brain 
Table 2: Distribution of Cav1 positivity in primary tumors and brain metastases

\begin{tabular}{|l|l|l|l|l|}
\hline \multirow{2}{*}{ Primary tumor } & \multicolumn{2}{|l|}{ Metastasis } & Sum & $\boldsymbol{p}$ \\
\cline { 2 - 4 } & Cav1 negative & Cav1 positive & & \\
\hline Cav1 negative & 39 & 14 & 53 & \multirow{2}{*}{$<0.001$} \\
\hline Cav1 positive & 2 & 14 & 16 & \\
\hline Sum & 41 & 28 & 69 & \\
\hline
\end{tabular}

neuroendocrine tumors ( 7 cases) were negative for Cav1 expression. Analysis of the differential expression of Cav1 by histotype revealed that all of the positive primary adenocarcinomas ( 7 cases) maintained Cav1 expression at the brain metastatic site and that 7 out of 31 acquired Cav1 expression during metastasis (chi-square test $p<0.001$ )

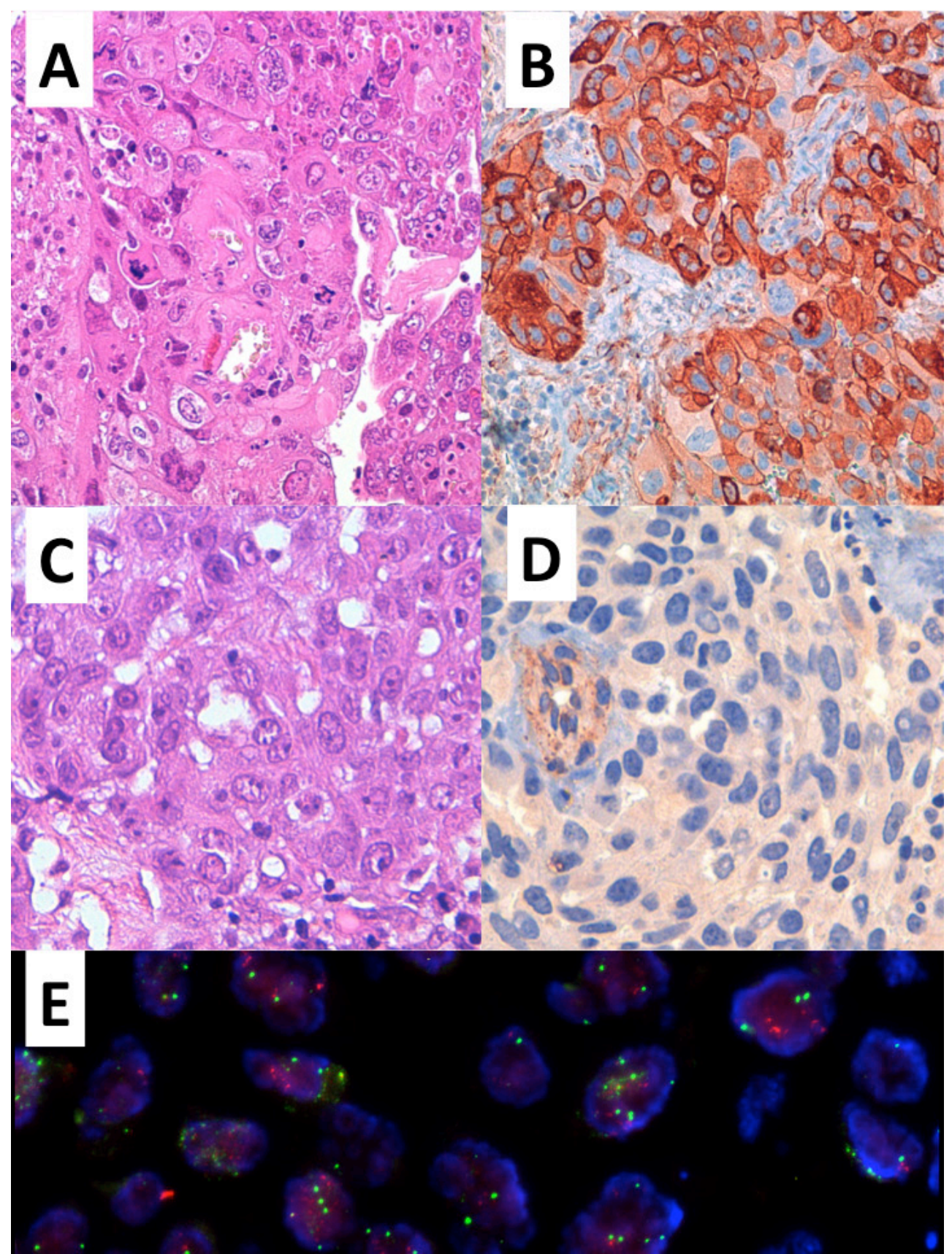

Figure 1: Representative examples of two brain metastasis from lung adenocarcinoma (A, C hematoxylin \& Eosin, original magnification $400 \mathrm{X}$ ) with mild to strong membrane-cytoplasmic immunoreactivity and negative staining for Cav1 (respectively B, D original magnification 400X) and high CAV1 gene polysomy by FISH analysis (E). 
(Table 3). No association of Cav1 expression was found for squamous cell or large cell carcinoma histotype (Table 4).

\section{Cav1 mutation and FISH analyses}

Fifty-four cases were suitable for mutational
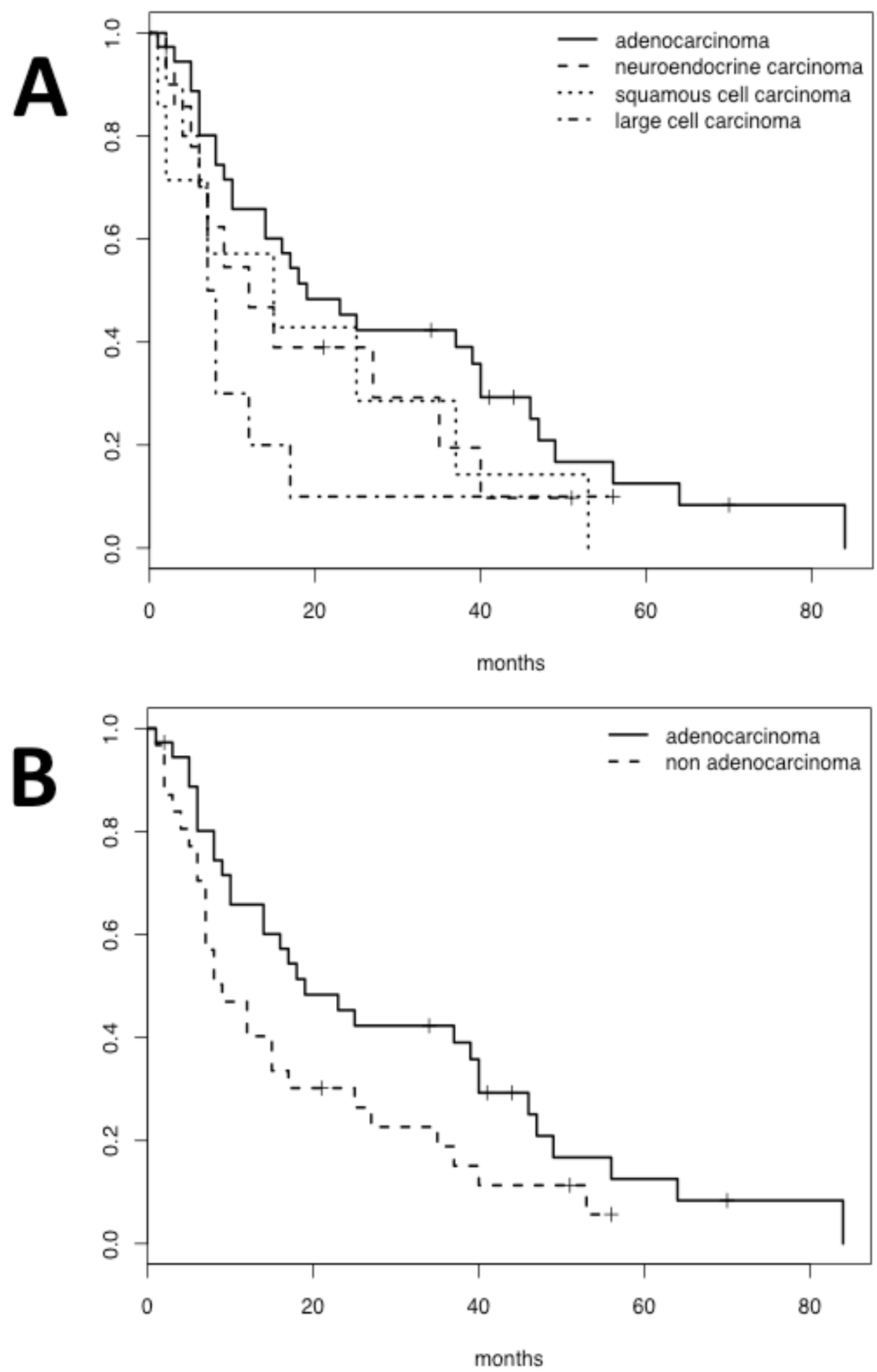

Figure 2: Survival curves showing that grouping by histotype was not a significant predictor for survival (Log rank test, $\mathbf{p}=\mathbf{0 . 2 3 3}, \mathbf{2 A})$. Conversely, adenocarcinoma was associated with prolonged survival when other histotypes were considered together (Log rank test, $\mathrm{p}=0.056,2 \mathrm{~B}$ ). 
Table 3: Differential expression of Cav1 by histotype and site

\begin{tabular}{|c|c|c|c|c|c|c|}
\hline & \multicolumn{2}{|c|}{ Primary lung cancer } & \multirow[b]{2}{*}{$p$} & \multicolumn{2}{|c|}{ Brain metastasis } & \multirow[b]{2}{*}{$p$} \\
\hline & Cav1 negative & Cav1 positive & & Cav1 negative & Cav1 positive & \\
\hline $\begin{array}{l}\text { Adenocarcinoma } \\
\text { Squamous cell } \\
\text { Neuroendocrine } \\
\text { Large cell }\end{array}$ & $\begin{array}{l}31 \\
9 \\
7 \\
6\end{array}$ & $\begin{array}{l}7 \\
5 \\
0 \\
4\end{array}$ & 0.143 & $\begin{array}{l}24 \\
6 \\
7 \\
4\end{array}$ & $\begin{array}{l}14 \\
8 \\
0 \\
6\end{array}$ & 0.043 \\
\hline
\end{tabular}

Table 4: Correlation between Cav1 expression at primary and metastatic sites in adenocarcinoma histotype

\begin{tabular}{|l|l|l|l|l|}
\hline & Primary tumor & Metastasis & $p$ \\
\cline { 3 - 5 } & adenocarcinoma & Cav1 negative & Cav1 positive & \\
\hline Adenocarcinoma & Cav1 negative & 24 & 7 & \multirow{2}{*}{$p<0.001$} \\
\hline & Cav1 positive & 0 & 7 & \multirow{2}{*}{1} \\
\hline Squamous cell & Cav1 negative & 4 & 5 & not \\
& Cav1 positive & 2 & 3 & available \\
\hline Neuroendocrine & Cav1 negative & 7 & 0 & \multirow{2}{*}{0.1472} \\
& Cav1 positive & 0 & 0 & \\
\hline \multirow{2}{*}{ Large cell } & Cav1 negative & 4 & 2 & \\
& Cav1 positive & 0 & 4 & \\
\hline
\end{tabular}

analysis. The Cav1 P132L mutation was found in two lung adenocarcinomas, only one of which retained the mutation at the metastatic site. Interestingly, this case had a negative immunohistochemistry result, whereas the other case showed positive results at the primary and secondary sites. FISH analysis was performed on 16 matched cases,

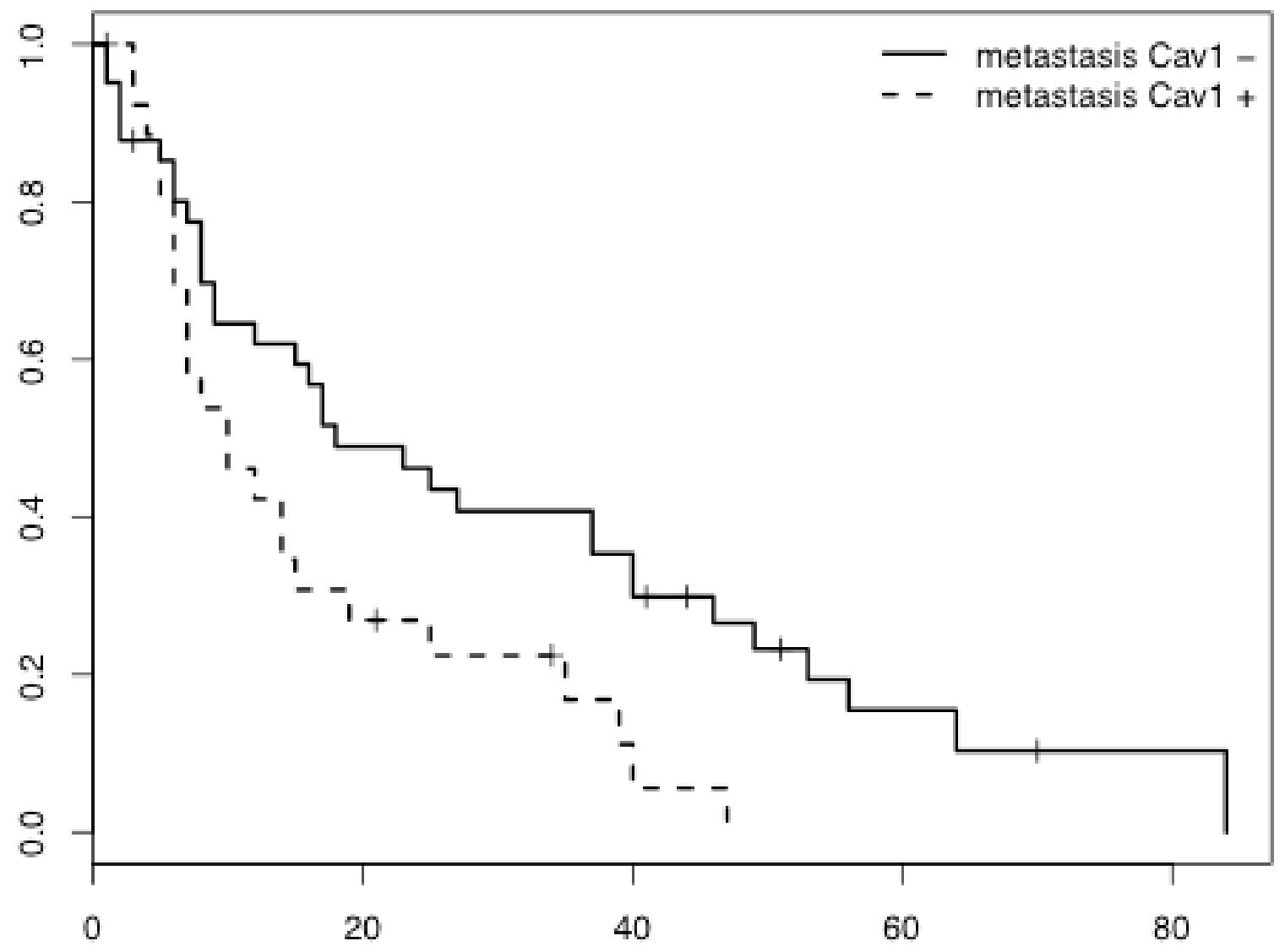

months

Figure 3: Survival curves demonstrating the association between Cav1 expression in brain metastasis and poor prognosis $(\log$ rank test, $p=0.015)$. 
Table 5: Proportional hazard model for the whole data set

\begin{tabular}{|l|l|l|l|l|}
\hline & Without interaction (a) & With interaction (b) \\
\hline & HR & $\boldsymbol{p}$-value & HR & \multicolumn{2}{|l|}{$\boldsymbol{p}$-value } \\
\hline Age & 1.061 & $\mathbf{0 . 0 0 2}$ & -- & -- \\
\hline Old age & -- & -- & 2.430 & $\mathbf{0 . 0 2 6}$ \\
\hline Histotype & 0.521 & $\mathbf{0 . 0 2 0}$ & 0.522 & $\mathbf{0 . 0 2 1}$ \\
\hline Cav1 positive metastasis & 2.359 & $\mathbf{0 . 0 0 4}$ & 2.571 & $\mathbf{0 . 0 3 1}$ \\
\hline Interaction effect Cav1/age & -- & -- & 1.183 & $\mathbf{0 . 7 8 0}$ \\
\hline
\end{tabular}

Table 6: Proportional hazard models restricted to radio-treated patients

\begin{tabular}{|l|l|l|l|l|}
\hline & \multicolumn{2}{l|}{ Without interaction (a) } & \multicolumn{2}{l|}{ With interaction (b) } \\
\hline & HR & $\boldsymbol{p}$-value & HR & $\boldsymbol{p}$-value \\
\hline Age & 1.059 & 0.018 & 1.069 & 0.008 \\
\hline Histotype adenocarcinoma & 0.388 & 0.004 & 0.321 & $<0.001$ \\
\hline Cav1-positive metastasis & 3.197 & 0.003 & 1.197 & 0.720 \\
\hline Radiotherapy & 1.034 & 0.920 & 0.411 & 0.041 \\
\hline Interaction effect Cav1/Radiotherapy & -- & -- & 6.839 & 0.004 \\
\hline
\end{tabular}

among which six displayed a gain and three exhibited amplification of Cav1 expression at both the primary and metastatic sites. The presence of a gain or amplification of expression in the primary neoplasm was significantly associated with the same event in the metastasis (Fisher's exact test $p=0.01$ ). None of the cases acquired a gain or amplification in the metastatic lesion. The two mutated cases showed neither a gain nor amplification.

\section{Survival analysis of Cav1 expression and responsiveness to radiotherapy}

Follow-up data, ranging from 1 to 84 months (median overall survival: 14 months), were available for all but one patient. At the time of analysis, 57 patients (84\%) had died, all because of brain metastasis. Among the $11(16 \%)$ who were living, two were Cav1 positive in both the primary lung lesion and brain metastasis, whereas one had acquired Cav1 expression in the metastasis. Overall, although grouping by histotype did not reveal differences in survival (log rank test, $p=0.233$, Figure $2 \mathrm{~A})$, the adenocarcinoma histotype was associated with prolonged survival (log rank test, $p=0.056$, median survival of 19 vs. 9 months for cases with adenocarcinoma and with other histotypes considered together, respectively, Figure 2B). Only Cav1 expression in the brain metastasis (and not its expression in the primary lesion) was related to poor prognosis (log rank test, $p=0.015$, median survival of 18 vs. 10 months for Cav1 negative and positive cases, respectively) (Figure 3). The presence of multiple metastases vs. a single brain metastasis (20 and 49 cases, respectively) was not associated with a significant difference in survival (log rank test, $p=0.999$ ). With a proportional hazard model controlling for age at diagnosis $(\mathrm{HR}=1.061,95 \% \mathrm{CI} 1.022$ to 1.101 , Wald test $p=0.002)$ and the adenocarcinoma histotype $(\mathrm{HR}=0.521,95 \% \mathrm{CI}$
0.301 to 0.901 , Wald test $p=0.020$ ), Cav1 expression in the metastasis (HR $=2.359,95 \%$ CI 1.309 to 4.251 , Wald test $p=0.004$ ) was associated with an increased risk of death, demonstrating its value as an independent predictor (Table 5a). To study the interaction between age and Cav1 expression at the metastatic site, we considered age as a factor, establishing "young" (age below the median of 63) and "old" (age $\geq 64$ ) classifications. Interestingly, Cav1positive metastasis was associated with an equivalent increase in the risk of death in the two age groups (interaction term, Wald test $p=0.780$ ) and neutralized the favorable effect of young age on survival compared with the older patients (old Cav1 negative $v s$. young Cav1 positive, $\mathrm{HR}=1.058,95 \% \mathrm{CI} 0.537$ to 2.083 , Wald test $p$ $=0.870)$ (Table 5b).

Evaluation of the patients with available brain radiotherapy data (49 patients, 44 deaths), controlling for age at diagnosis, the adenocarcinoma histotype and Cav1 expression in the brain metastasis, revealed that radiotherapy was not significantly associated with survival (Table 6a). However, when accounting for an interaction between Cav1 expression and radiotherapy, the treatment was associated with a reduced risk of death in the patients with Cav1-negative brain metastasis (17 out of 27 cases, $\mathrm{HR}=0.411,95 \%$ CI 0.175 to 0.966 , Wald test $p=0.041$ ) and with an increased risk of death in those with Cav1positive brain metastasis ( 14 out of 22 cases, $\mathrm{HR}=6.839$, $95 \%$ CI 1.849 to 25.301 , Wald test $p=0.004$ ) (Table $6 \mathrm{~b}$ ). In this subgroup of radiotherapy-treated patients, wholebrain radiotherapy was administered with adjuvant intent to 22 patients with a single surgically removed lesion and with palliative-cytoreductive intent to the remaining 9 patients with multiple brain lesions. Table 7 reports an extension of analysis using the proportional hazard model, accounting for radiotherapy intent (adjuvant or cytoreductive). The hazard ratios were found to be similar for the adjuvant and cytoreductive intents $(\mathrm{HR}=0.383$ 
Table 7: Proportional hazard model differentiating for adjuvant or palliative-cytoreductive radiotherapy treatment

\begin{tabular}{|l|l|l|}
\hline & HR & p-value \\
\hline Age & 1.067 & $\mathbf{0 . 0 1 4}$ \\
\hline Histotype adenocarcinoma & 0.312 & $<\mathbf{0 . 0 0 1}$ \\
\hline Cav1-positive metastasis & 1.180 & 0.740 \\
\hline Adjuvant Radiotherapy & 0.383 & $\mathbf{0 . 0 5 0}$ \\
\hline Palliative-cytoreductive Radiotherapy & 0.475 & 0.210 \\
\hline Interaction effect Cav1/ Adjuvant Radiotherapy & 7.177 & $\mathbf{0 . 0 0 7}$ \\
\hline Interaction effect Cav1/ Cytoreductive Radiotherapy & 6.534 & $\mathbf{0 . 0 4 3}$ \\
\hline
\end{tabular}

vs. $\mathrm{HR}=0.475$, respectively) and likewise for the two interaction terms (Cav1/adjuvant $\mathrm{HR}=7.17$ vs. Cav1/ cytoreductive HR =6.534). Model selection based on loglikelihood ratio tests $(p$-value $=0.934)$ favored the model with interactions that did not account for the radiotherapy intent (Table 6b).

\section{DISCUSSION}

In this study, we found that Cav1 expression in resected brain metastasis from NSCLC is a reliable predictor of poor survival and an independent predictor of poor radiotherapy responsiveness.

Prognostic role of adenocarcinoma histotype in brain metastasis: Lung cancer is the most frequent site of origin for brain metastasis, which is diagnosed in approximately $20-30 \%$ of patients during the course of their disease [2]. Approximately half of patients have a single brain metastasis [20]. Brain metastasis is more frequently found in NSCLC patients [21, 22], and these patients are usually considered terminal and to have poor prognosis. According to current guidelines $[23,24]$, resection of solitary brain metastasis is a valuable treatment option, resulting in a five-year survival rate of $20-25 \%$ if radical surgery of the primary tumor is also performed [25]. In agreement with a recent systematic review [26], survival improves when both the primary lung tumor and brain metastasis are resected; thus, removal of the primary site and single brain metastasis appears to be an effective treatment for long-term survival. However, effective tools for selecting patients who may benefit from surgical resection of brain metastasis remain to be identified. With respect to systemic therapy, tumor features, such as the histological subtype and molecular signature, play relevant prognostic and predictive roles because different NSCLC histologies have different treatment approaches and prognoses [27]. Surgical resection of brain metastasis from lung squamous cell carcinoma results in a significantly worse survival rate compared with other histotypes, whereas patients with adenocarcinoma live significantly longer [28]. Consistent with these findings, we found that the adenocarcinoma histotype is associated with prolonged survival, and advanced age is linked with poor prognosis.

Prognostic role of Cav1 expression in brain metastasis: Expression of Cav1 in human cancer cell lines and tumor samples has been documented in numerous studies, and it is increasingly clear that its role depends on the tumor type and stage [29]. In advanced stages, Cav1 gains an oncogenic role because its increased level favors the development of cellular traits associated with enhanced malignancy, including multidrug resistance and metastasis [30]. Accordingly, high Cav1 expression is a negative prognostic factor for overall and disease-free survival in patients with various tumor types, including breast [31], esophagus [32], pancreas [33], kidney [34], and prostate tumors [35], meningioma [36] and oligodendroglioma [37]. Concerning lung cancer, normal human lung epithelial cultures abundantly express Cav1, whereas its expression is reduced or absent in $95 \%$ of small-cell lung cancer cell lines and is retained in $76 \%$ of NSCLC cells [15]. In NSCLC cell lines, Cav1 expression increases anoikis resistance, migration and invasiveness [15, 3840]. Anoikis-resistant NSCLC cells overexpressing Cav1 exhibit significant increases in anchorage-independent growth, extracellular matrix adhesion, migration, and invasion, whereas Cav1 knockdown by shRNA transfection is able to reverse the metastatic potential [16]. The findings of the present study are consistent with those of our previous study of differential Cav1 expression according to histotype [18], showing that all lung large cell neuroendocrine carcinomas and matched brain metastases were Cav1 negative. In addition, 20\% of the primary tumors shifted to Cav1 positivity during metastasis. Many studies, including a recent metaanalysis [17], have confirmed that Cav1 tissue expression is a negative prognostic marker in NSCLC and that it is correlated with differentiation, $\mathrm{T}$ stage and lymph node metastasis. However, the clinical significance of Cav1 expression in resected brain metastasis has not yet been investigated. In our study, only Cav1 expression in brain metastasis, and not in the primary tumor, was correlated with poor prognosis and an increased risk of death in the multivariate model, demonstrating its value as an independent prognosticator. Moreover, as age is per se an adverse prognostic factor, we investigated the relationship between age and Cav1 expression at the metastatic site, demonstrating that positive Cav1 metastasis was independently associated with a higher risk of death, exclusively in the group of young patients.

A somatic missense mutation in $C A V 1$ at codon 
132, converting proline to leucine (P132L), has been detected in $11-16 \%$ of invasive breast cancer patients $[41,42]$. As the recombinant expression of the CAV1 P132L mutant leads to mislocalization and intracellular retention of wild-type $C A V 1$, cellular transformation, and promotion of invasive ability [41], a dominant-negative behavior has been suggested for the P132L mutant [41, 43]. Although this mutation was not identified in any of 46 lung cancer cell lines examined by Sunaga et al. [15] and has not been described in other studies investigating mutations in lung cancer $[38,44]$, we detected it in two human adenocarcinoma specimens. Both patients with this CAV1 P132L mutation had a single brain metastasis, no extracranial tumors and a Karnofsky Performance Status (KPS) of 90. Their survival times (47 and 49 months) were longer than the median survival time of the series (14 months). These findings indicate that mutation of $C A V I$ results in a loss of its unfavorable prognostic/predictive value, in contrast with what has been previously described in different oncologic contexts [43].

Predictive role of Cav1 for radiotherapy responsiveness in brain metastasis: In solitary brain metastasis, surgical resection followed by WBRT or stereotactic radiosurgery might be beneficial [2]. However, the adjuvant treatment of these patients still results in disappointing outcomes because WBRT only extends survival by three to six months, even when achieving palliative improvement of neurological symptoms [3]. In our series, radiotherapy was not significantly associated with survival. Nevertheless, among the radiotherapytreated patients, independent of the intent, Cav1 proved to be a useful marker for predicting radiotherapy responsiveness because the patients with Cav1-negative brain metastasis had a reduced risk of death, whereas those with Cav1 positivity showed an increased risk. These findings are consistent with reports of induction of radiosensitization by Cav-1 knockdown in the same tumor model $[10,11]$ and radiation-elicited Cav1 expression in pancreatic cancer cell lines [10]. Moreover, low Cav1 expression in pre-treatment biopsies from patients with locally advanced colorectal cancer has been correlated with better rates of local control and overall survival [12].

\section{CONCLUSIONS}

Cav1 expression in brain metastasis from lung cancer is independently predictive of worse outcome and radioresistance. Therefore, in the future development of personalized therapy for advanced oncologic patients, Cav1 could become an additional tool to prognosticate and predict a distinguished subset of patients with brain metastatic lung cancer.

\section{MATERIALS AND METHODS}

\section{Case collection}

Sixty-nine NSCLCs and their matched resected brain metastases were retrieved from consecutive cohorts from the archives of two different institutions, the Pathology Divisions of the University of Torino at Città della Salute e della Scienza (Molinette) Hospital in Turin and San Luigi Hospital in Orbassano, Italy. In 25 cases $(36 \%)$, brain metastasis was diagnosed synchronously with primary NSCLC, and in three cases (4\%), brain metastasis was diagnosed prior to primary lung cancer (1 to 3 months before). In the remaining 41 cases (64\%), metastasis was metachronous (mean of 9 months from NSCLC diagnosis, range 1-63). None of the patients had extra-cranial tumors. At the time of neurosurgical resection, the patients with a single brain metastasis comprised $71 \%$ of the total patients (49 patients), whereas the remaining $29 \%$ of the patients (20 patients) had multiple metastases. The patients with a single brain metastasis underwent conventional surgery. Thirteen out of 69 patients received chemotherapy. Information about brain radiotherapy was available for 49 patients. Among them, 22 patients with a single surgically removed lesion received radiotherapy with an adjuvant intent; conversely, nine patients with multiple brain lesions underwent radiotherapy with a palliative-cytoreductive intent. Whole-brain radiotherapy was administered with the same schedule (30 Gy in 10 fractions) in both patient groups. The group of patients treated with adjuvant radiotherapy had a good clinical performance status (mean KPS of 90) without any significant neurological deficits. The patients treated with cytoreductive whole-brain radiotherapy for active metastatic disease occasionally had some neurological deficits related to the site of the disease, but the KPS did not fall lower than 70, which is mandatory for irradiation. Similarly, all of the patients who did not receive radiotherapy had a KPS of over 70 . All of the case data were anonymously recorded. The study received ethical approval from the local Institutional Review Boards.

\section{Histopathological evaluation}

All hematoxylin and eosin (H\&E)-stained slides available from surgical specimens were reviewed, and a representative paraffin block was selected for each case. The primary tumor and matched brain metastasis from each patient were independently reviewed by two dedicated pathologists (RS and PC), who confirmed all diagnoses according to the current World Health Organization classification [19]. 


\section{Immunohistochemistry}

Immunohistochemistry was performed in all cases. Three micrometer-thick serial paraffin sections for each case were processed by immunohistochemistry using an antibody against Cav1 (Santa Cruz, Santa Cruz, CA, USA, rabbit polyclonal, diluted 1/350) with an automated immunostainer (Ventana BenchMark Auto-Stainer, Ventana Medical Systems). A biotin-free, dextran chainbased detection system (EnVysion, Dako, Carpinteria, CA, USA) was used for visualization, and diaminobenzidine (Ventana Medical Systems, Tucson, AZ, USA) was used as a chromogen, according to standard protocols. Vascular endothelium represented an internal positive control.

\section{Staining interpretation and scoring system}

All immunostained slides were analyzed independently by RS, ED and PC, who were blinded to the clinical data. Cav1 staining was assessed as a categorical variable (negative or positive if present in at least $10 \%$ of neoplastic cells). In cases of discrepancies, slides were reviewed using a multihead microscope, and a consensus was reached.

\section{Mutational analysis}

Mutational analysis of the Cav1 gene was performed in 54 cases (in both the primary tumor and metastasis) to identify somatic mutations. Genomic DNA extractions were performed with standard methods (NucleoSpin Tissue Kit, Macherey-Nagel, Duren, Germany), and 6 ng of DNA was amplified using specific primers for the human Cav1 gene. Specifically, PCR primers were designed to amplify and sequence exon 2 and exon 3 of Cav 1 as follows: Cav1F-exon2: 5'-GCAGGGACATCTCTACACG-3'; Cav-1Rexon2: 5'-GCCTTGGCTTACCTTGACCA-3'; Cav1Fexon3: 5'-AACCAGAAGGGACACACAG-3'; and Cav-1R-exon3: 5'-AAAGAGTGGGTCACAGACG-3'.

The somatic origin of each mutation was confirmed by the sequencing of both neoplastic and normal DNA obtained by laser-capture microdissection of a nontransformed area adjacent to the neoplastic lesion. For small samples, normal DNA was extracted from a different histological specimen collected from the same patient. Mutations were detected only in malignant tissues. Positive results for mutational analysis were validated at least twice in independent PCRs. Pathologists carrying out these analyses were blinded to the clinical outcomes of the patients.

\section{Cav-1 fluorescence in situ hybridization (FISH) analysis}

FISH was performed using both an alpha satellite probe specific for chromosome 7 (CEP7) directly labeled with green fluorochrome (Cytocell Technologies Ltd., UK) as a control probe and a self-made probe specific for the CAV1,2 locus, BAC 688K20 (7q31.2). The clone was obtained from Invitrogen (Invitrogen Corporation, USA). UCSC database (http://genome.ucsc.edu July 2008 release) was queried for the localization of the probe. The BAC was expanded, extracted and then directly labeled with SpectrumOrange-dUTP (Abbott Molecular, Europe) using a BioPrime DNA Labeling System (Invitrogen Corporation, USA), according to the manufacturer's instructions. To analyze the position and strength of the signal, the presence/absence of background and crosshybridization, and the hybridization efficiency, the $\mathrm{BAC}$ clone was tested using metaphase and interphase cells from healthy donors obtained using conventional cytogenetic methods. FISH was routinely performed with the two probes CAV1,2 and CEP7 on formalinfixed, paraffin-embedded tissue. Images of red $(\mathrm{CAV} 1,2)$ and green (CEP7) spots in areas with significant signal were automatically acquired using 13 focus planes, with Metafer software by a MetaSystem scanning station (Carl Zeiss MetaSystems GmbH, Altlussheim, Germany) equipped with an AxioImager epifluorescence microscope. The automatically acquired images were analyzed with Isis software (Zeiss). Eighty to 100 non-overlapping neoplastic nuclei with two control green signals $(2 \mathrm{G})$ were analyzed for each case and were scored as follows: $2 \mathrm{R}$, normal nuclei; R > 3, CAV gain; R > 5, CAV amplification; and $\mathrm{R}<2$, CAV loss.

\section{Statistical analysis}

Correlations between Cav1 expression and the clinico-pathological variables were quantified using the chi-square test or Fisher's exact test, as appropriate. Kaplan-Meier curves and the log rank test were used to assess differences in survival between the groups of patients. Overall survival was defined as the time elapsed from the date of lung cancer diagnosis to the date of death or the last visit. Hazard ratios (HRs) and 95\% confidence intervals (CIs) were estimated by the Cox proportional hazards model for multivariate survival analysis. The loglikelihood ratio test based on a chi-square approximation was used to perform model selection as appropriate. Statistical significance was set at a level of 0.05. All analyses were conducted using version 2.12 .1 of $\mathrm{R}$ statistical package (www.r-project.org). 


\section{GRANT SUPPORT}

This paper was supported by grants from FIRC (Fondazione Italiana per la Ricerca sul Cancro) to RS and from "Fondi di Ricerca Locale ex-60\%, 2013" to PC.

\section{CONFLICTS OF INTEREST}

The authors declare that they have no conflicts of interest.

\section{REFERENCES}

1. Barnholtz-Sloan JS, Sloan AE, Davis FG, Vigneau FD, Lai $\mathrm{P}$ and Sawaya RE. Incidence proportions of brain metastases in patients diagnosed (1973 to 2001) in the Metropolitan Detroit Cancer Surveillance System. J Clin Oncol. 2004; 22:2865-2872.

2. Besse B, Adjei A, Baas P, Meldgaard P, Nicolson M, PazAres L, Reck M, Smit EF, Syrigos K, Stahel R, Felip E, Peters $\mathrm{S}$ and Panel M. 2nd ESMO Consensus Conference on Lung Cancer: non-small-cell lung cancer first-line/ second and further lines of treatment in advanced disease. Ann Oncol. 2014; 25:1475-1484.

3. Baykara M, Kurt G, Buyukberber S, Demirci U, Ceviker $\mathrm{N}$, Algin E, Coskun U, Aykol S, Emmez H, Ozet A and Benekli M. Management of brain metastases from nonsmall cell lung cancer. J Cancer Res Ther. 2014; 10:915921.

4. Soffietti R, Ruda R and Trevisan E. Brain metastases: current management and new developments. Curr Opin Oncol. 2008; 20:676-684.

5. Sperduto PW, Kased N, Roberge D, Xu Z, Shanley R, Luo X, Sneed PK, Chao ST, Weil RJ, Suh J, Bhatt A, Jensen AW, Brown PD, Shih HA, Kirkpatrick J, Gaspar LE, et al. Summary report on the graded prognostic assessment: an accurate and facile diagnosis-specific tool to estimate survival for patients with brain metastases. J Clin Oncol. 2012; 30:419-425.

6. Lin NU. Targeted therapies in brain metastases. Curr Treat Options Neurol. 2014; 16:276.

7. Yang CP, Galbiati F, Volonte D, Horwitz SB and Lisanti MP. Upregulation of caveolin-1 and caveolae organelles in Taxol-resistant A549 cells. FEBS Lett. 1998; 439:368-372.

8. Belanger MM, Gaudreau M, Roussel E and Couet J. Role of caveolin-1 in etoposide resistance development in A549 lung cancer cells. Cancer Biol Ther. 2004; 3:954-959.

9. Brodie SA, Lombardo C, Li G, Kowalski J, Gandhi K, You S, Khuri FR, Marcus A, Vertino PM and Brandes JC. Aberrant promoter methylation of caveolin-1 is associated with favorable response to taxane-platinum combination chemotherapy in advanced NSCLC. PLoS One. 2014; 9:e107124.

10. Cordes N, Frick S, Brunner TB, Pilarsky C, Grutzmann
R, Sipos B, Kloppel G, McKenna WG and Bernhard EJ. Human pancreatic tumor cells are sensitized to ionizing radiation by knockdown of caveolin-1. Oncogene. 2007; 26:6851-6862.

11. Hehlgans S, Eke I, Storch K, Haase M, Baretton GB and Cordes N. Caveolin-1 mediated radioresistance of 3D grown pancreatic cancer cells. Radiother Oncol. 2009; 92:362-370.

12. Rödel F, Capalbo G, Rödel C and Weiss C. Caveolin-1 as a prognostic marker for local control after preoperative chemoradiation therapy in rectal cancer. Int J Radiat Oncol Biol Phys 2009; 73:846-852.

13. Parat MO. The biology of caveolae: achievements and perspectives. Int Rev Cell Mol Biol. 2009; 273:117-162.

14. Senetta R, Stella G, Pozzi E, Sturli N, Massi D and Cassoni P. Caveolin-1 as a promoter of tumour spreading: when, how, where and why. J Cell Mol Med. 2013; 17:325-336.

15. Sunaga N, Miyajima K, Suzuki M, Sato M, White MA, Ramirez RD, Shay JW, Gazdar AF and Minna JD. Different roles for caveolin-1 in the development of non-small cell lung cancer versus small cell lung cancer. Cancer Res. 2004; 64:4277-4285.

16. Chanvorachote P, Pongrakhananon V and Halim H. Caveolin-1 regulates metastatic behaviors of anoikis resistant lung cancer cells. Mol Cell Biochem. 2015; 399:291-302.

17. Chen D, Shen C, Du H, Zhou Y and Che G. Duplex value of caveolin-1 in non-small cell lung cancer: a meta analysis. Fam Cancer. 2014; 13:449-457.

18. Cassoni P, Daniele L, Maldi E, Righi L, Tavaglione V, Novello S, Volante M, Scagliotti GV and Papotti M. Caveolin-1 expression in lung carcinoma varies according to tumour histotype and is acquired de novo in brain metastases. Histopathology. 2009; 55:20-27.

19. Travis WD, Brambilla E and Muller-Hermelink KM. (2015). WHO Classification of Tumours of the Lung, Pleura, Thymus and Heart. Lyon.

20. Gavrilovic IT and Posner JB. Brain metastases: epidemiology and pathophysiology. J Neurooncol. 2005; 75:5-14.

21. Varlotto JM, Flickinger JC, Niranjan A, Bhatnagar AK, Kondziolka D and Lunsford LD. Analysis of tumor control and toxicity in patients who have survived at least one year after radiosurgery for brain metastases. Int J Radiat Oncol Biol Phys. 2003; 57:452-464.

22. Chen AM, Jahan TM, Jablons DM, Garcia J and Larson DA. Risk of cerebral metastases and neurological death after pathological complete response to neoadjuvant therapy for locally advanced nonsmall-cell lung cancer: clinical implications for the subsequent management of the brain. Cancer. 2007; 109:1668-1675.

23. Ettinger DS, Wood DE, Akerley W, Bazhenova LA, Borghaei H, Camidge DR, Cheney RT, Chirieac LR, D'Amico TA, Demmy TL, Dilling TJ, Govindan R, Grannis 
FW, Jr., Horn L, Jahan TM, Komaki R, et al. Non-small cell lung cancer, version 1.2015. J Natl Compr Canc Netw. 2014; 12:1738-1761.

24. Reck M, Popat S, Reinmuth N, De Ruysscher D, Kerr KM, Peters S and Group EGW. Metastatic non-small-cell lung cancer (NSCLC): ESMO Clinical Practice Guidelines for diagnosis, treatment and follow-up. Ann Oncol. 2014; 25 Suppl 3:iii27-39.

25. Abrahams JM, Torchia M, Putt M, Kaiser LR and Judy KD. Risk factors affecting survival after brain metastases from non-small cell lung carcinoma: a follow-up study of 70 patients. J Neurosurg. 2001; 95:595-600.

26. Qin H, Wang C, Jiang Y, Zhang X, Zhang Y and Ruan Z. Patients with single brain metastasis from non-small cell lung cancer equally benefit from stereotactic radiosurgery and surgery: a systematic review. Med Sci Monit. 2015; 21:144-152.

27. Guo S, Reddy CA, Chao ST, Suh JH, Barnett GH, Vogelbaum MA and Videtic GM. Impact of non-small cell lung cancer histology on survival predicted from the graded prognostic assessment for patients with brain metastases. Lung Cancer. 2012; 77:389-393.

28. Putora PM, Ess S, Panje C, Hundsberger T, van Leyen K, Plasswilm L and Fruh M. Prognostic significance of histology after resection of brain metastases and whole brain radiotherapy in non-small cell lung cancer (NSCLC). Clin Exp Metastasis. 2015; 32:143-149.

29. Goetz JG, Lajoie P, Wiseman SM and Nabi IR. Caveolin-1 in tumor progression: the good, the bad and the ugly. Cancer Metastasis Rev. 2008; 27:715-735.

30. Nunez-Wehinger S, Ortiz RJ, Diaz N, Diaz J, LobosGonzalez L and Quest AF. Caveolin-1 in cell migration and metastasis. Curr Mol Med. 2014; 14:255-274.

31. Mercier I and Lisanti MP. Caveolin-1 and breast cancer: a new clinical perspective. Adv Exp Med Biol. 2012; 729:8394.

32. Ando T, Ishiguro H, Kimura M, Mitsui A, Mori Y, Sugito N, Tomoda K, Mori R, Harada K, Katada T, Ogawa R, Fujii Y and Kuwabara Y. The overexpression of caveolin-1 and caveolin-2 correlates with a poor prognosis and tumor progression in esophageal squamous cell carcinoma. Oncol Rep. 2007; 18:601-609.

33. Chen T, Liu L, Xu HX, Wang WQ, Wu CT, Yao WT and $\mathrm{Yu}$ XJ. Significance of caveolin-1 regulators in pancreatic cancer. Asian Pac J Cancer Prev. 2013; 14:4501-4507.

34. Campbell L, Jasani B, Edwards K, Gumbleton M and Griffiths DF. Combined expression of caveolin-1 and an activated AKT/mTOR pathway predicts reduced diseasefree survival in clinically confined renal cell carcinoma. $\mathrm{Br}$ J Cancer. 2008; 98:931-940.

35. Freeman MR, Yang W and Di Vizio D. Caveolin-1 and prostate cancer progression. Adv Exp Med Biol. 2012; 729:95-110.

36. Barresi V, Cerasoli S, Paioli G, Vitarelli E, Giuffre
G, Guiducci G, Tuccari G and Barresi G. Caveolin-1 in meningiomas: expression and clinico-pathological correlations. Acta Neuropathol. 2006; 112:617-626.

37. Senetta R, Trevisan E, Ruda R, Maldi E, Molinaro L, Lefranc F, Chiusa L, Lanotte M, Soffietti R and Cassoni P. Caveolin 1 expression independently predicts shorter survival in oligodendrogliomas. J Neuropathol Exp Neurol. 2009; 68:425-431.

38. Ho CC, Huang PH, Huang HY, Chen YH, Yang PC and Hsu SM. Up-regulated caveolin-1 accentuates the metastasis capability of lung adenocarcinoma by inducing filopodia formation. Am J Pathol. 2002; 161:1647-1656.

39. Chanvorachote P, Nimmannit U, Lu Y, Talbott S, Jiang BH and Rojanasakul Y. Nitric oxide regulates lung carcinoma cell anoikis through inhibition of ubiquitin-proteasomal degradation of caveolin-1. J Biol Chem. 2009; 284:2847628484.

40. Rungtabnapa P, Nimmannit U, Halim H, Rojanasakul Y and Chanvorachote P. Hydrogen peroxide inhibits nonsmall cell lung cancer cell anoikis through the inhibition of caveolin-1 degradation. Am J Physiol Cell Physiol. 2011; 300:C235-245.

41. Hayashi K, Matsuda S, Machida K, Yamamoto T, Fukuda Y, Nimura Y, Hayakawa T and Hamaguchi M. Invasion activating caveolin-1 mutation in human scirrhous breast cancers. Cancer Res. 2001; 61:2361-2364.

42. Li T, Sotgia F, Vuolo MA, Li M, Yang WC, Pestell RG, Sparano JA and Lisanti MP. Caveolin-1 mutations in human breast cancer: functional association with estrogen receptor alpha-positive status. Am J Pathol. 2006; 168:1998-2013.

43. Lee H, Park DS, Razani B, Russell RG, Pestell RG and Lisanti MP. Caveolin-1 mutations (P132L and null) and the pathogenesis of breast cancer: caveolin-1 (P132L) behaves in a dominant-negative manner and caveolin-1 (-/-) null mice show mammary epithelial cell hyperplasia. Am J Pathol. 2002; 161:1357-1369.

44. Wikman H, Kettunen E, Seppanen JK, Karjalainen A, Hollmen J, Anttila S and Knuutila S. Identification of differentially expressed genes in pulmonary adenocarcinoma by using cDNA array. Oncogene. 2002; 21:5804-5813. 